\title{
A phenomenological view of Fermi-INTEGRAL gamma-ray Blazars, in the framework of leptonic SSC and EC scenario
}

\author{
Andrea Tramacere* \\ ISDC, Data Centre for Astrophysics Chemin d'Ecogia 16 CH-1290 Versoix Switzerland \\ E-mail: andrea.tramacere@unige.ch
}

\section{Claudio Ricci \& Roland Walter}

ISDC, Data Centre for Astrophysics Chemin d'Ecogia 16 CH-1290 Versoix Switzerland

\begin{abstract}
We propose a phenomenological approach that uses the spectral features of the rising part of the Inverse Compton emission of the blazars Spectral Energy Distribution, to derive the low-energy branch of the energy distribution of the emitting particles. We base our analysis on a leptonic, single-zone, homogeneous, Synchrotron Self-Compton, and External Compton scenario. Our analysis shows that is possible to use the values of photon index $\left(\alpha_{X}\right)$ observed in INTEGRAL Flat Spectrum Radio Quasars (FSRQs) and intermediate/low-peaked BL Lacs (I/LBLs), and the photon index $\left(\alpha_{\gamma}\right)$ observed in Fermi-LAT high-peaked BL Lacs (HBLs), to constrain the low-energy cut-off $\left(\gamma_{\min }\right)$, and the low-energy photon index $(s)$, of the emitting particles energy distribution. We found, in the case of FSRQSs and I/LBL Lacs, that INTEGRAL data rule out a scenario with $\gamma_{\min }>>1$. In the case of HBLs, Fermi data hints for a scenario with $\gamma_{\min }<<10^{4}$, in agreement with previous analysis of the HBL Mrk 421, based on simultaneous X-ray-to-UV data. The predicted ranges for $s$, derived from observed data, are $\simeq$ [1.6 -2.4], and $\simeq[1.2-2.8]$, respectively for HBLs, and FSRQs and I/LBLs. The similarity in the range of the predicted values of $s$, and the same trend of the position of the low-energy cut-off of the electron distribution, observed in FSRQs and BL Lacs, are interesting features, hinting for a common acceleration scenario acting in the two classes of objects.
\end{abstract}

8th INTEGRAL Workshop The Restless Gamma-ray Universe September 27-30, 2010

Dublin Ireland

\footnotetext{
* Speaker.
} 


\section{Introduction}

Blazars are Active Galactic Nuclei (AGNs) characterized by a polarised and highly variable non-thermal continuum emission extending from radio to $\gamma$-rays. In the most accepted scenario, this radiation is produced within a relativistic jet that originates in the central engine and points close to our line of sight. Blazars come in two main flavours: BL Lac objects and Flat Spectrum Radio Quasars (FSRQs). The former type is characterised by featureless optical spectra and their SEDs are usually interpreted in the framework of pure synchrotron self Compton model (SSC) (Jones et al., 1974; Ghisellini \& Maraschi, 1989) . On the contrary, FSRQs display the prominent emission lines that are typical of QSOs, and are likely to have the inverse Compton (IC) component dominated by the external radiation Compton (EC) emission (Sikora et al., 1994; Dermer \& Schlickeiser, 2002). BL Lac objects are often subdivided into three subclasses depending on their SEDs. This classification (Padovani \& Giommi, 1995) uses the peak energy of the synchrotron emission, which reflects the maximum energy the particles can be accelerated in the jet, to classify BL Lacs into low energy, intermediate energy, and high energy synchrotron peak objects, respectively called LBL, IBL, and HBL. The broad band spectral energy distribution of Blazars depends strongly on the shape of the underlying emitting particle distribution. Although radiative processes are quite well understood, the acceleration processes are still not completely understood, and several possible mechanisms are likely to explain the observed phenomenology. This degeneracy is due to the lack of a complete constraining of the shape of the emitting particle distribution. In particular, it is often difficult to find direct observational signatures to constrain the low energy branch of the emitting particle distribution, namely the lowest energy of the emitting particle distribution $\left(\gamma_{\min }\right)$ and its spectral slope $(s)$. We propose a phenomenological approach that uses the spectral features of the rising part of the IC component of blazars, to derive $\gamma_{\min }$ and $s$. In this regard, we selected the sample of FSQRs and I'LBLs already detected by INTEGRAL and Fermi-LAT, and the sample of HBLs detected by Fermi-LAT (Abdo et al., 2010). The INTEGRAL sample consists of all the blazars detected by INTEGRAL IBIS/ISGRI (Ubertiniet al., 2003) during its first 8 years of operations. The INTEGRAL spectra were extracted in the 17-150 keV range from an all-sky mosaic, created using all the public science windows as of May 2010 (see for details Ricciet al. (2011)).We decide to study FSRQs and I/LBLs in the INTEGRAL hard-X-ray windows because, for these objects, the INTEGRAL/ISGRI instrument is sampling the rising part of the IC SED, at frequencies where are emitting low energy particles. This allows us to use these spectral features to investigate the low energy branch of the electron distribution. Since we are dealing with low-energy particles, these particle are not cooling dominated, introducing in our estimates a low cooling contamination to the accelerative signature. A parallel scenario applies if we investigate the rising part of the HBLs IC emission through the Fermi-LAT spectral window.

\section{Phenomenological investigation of the low-energy branch of the electron distribution}

Our analysis relies on the study of the dependence of the rising part of the IC emission on the low-energy branch of the electron distribution. Our goal is to derive $\gamma_{\min }$ and $s$ from the photon index $\left(\alpha_{X}\right)$ observed by INTEGRAL/ISGRI (in the case of FSRQs and I/IBLs), and to derive the 

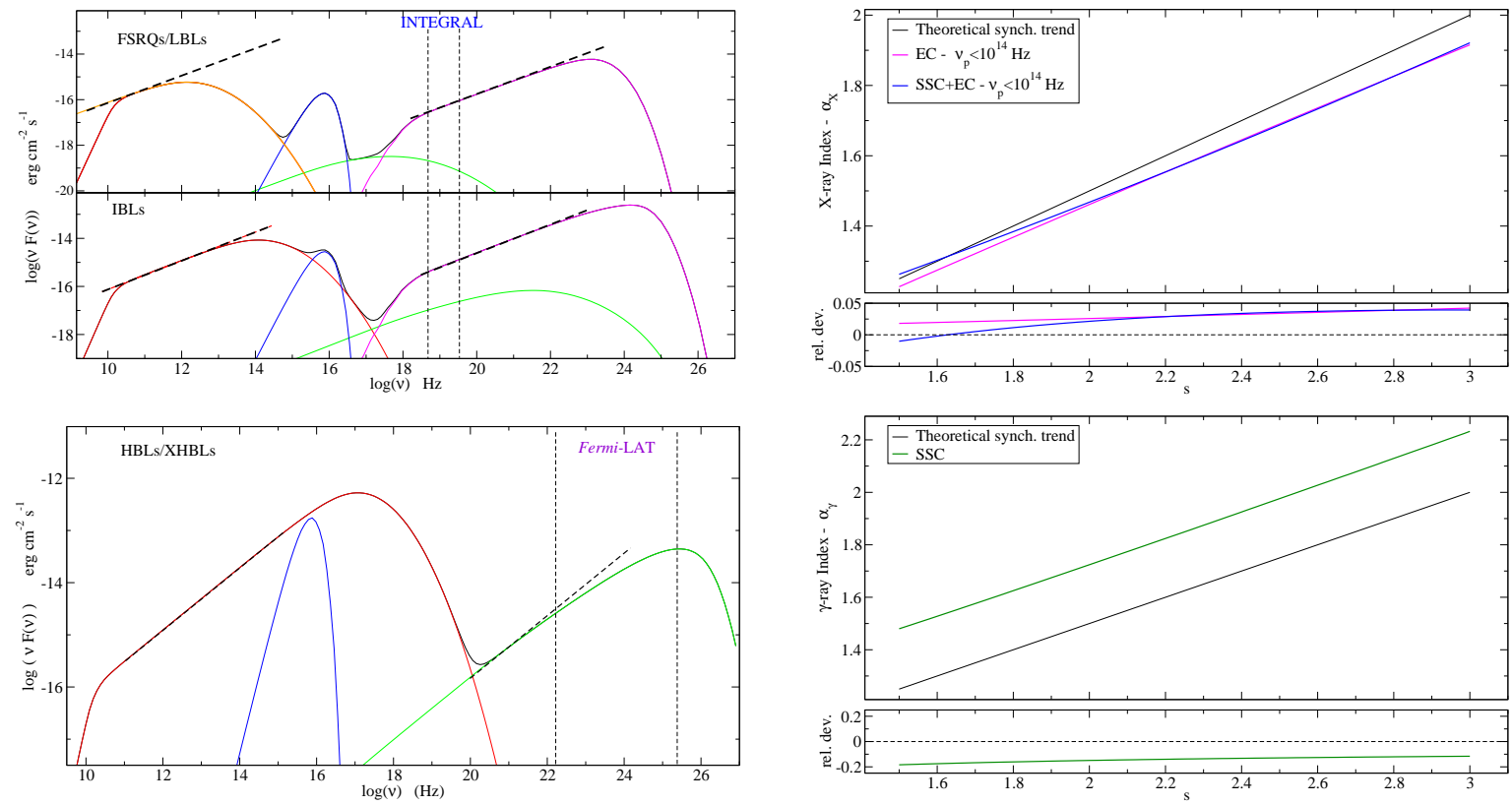

Figure 1: Left panels: typical SEDs for FSRQs/LBLs/IBLs (upper panel) and HBLs (panel $b$ ), the model shows: the synchrotron component (red line: with self-absorption, orange: without self-absorption), the SSC component (green line), the EC emission (purple line), the BBB emission (blue line), and the dashed black line represent the sum of all the components. The dashed thick oblique black line shows the approximate spectral slope. The vertical dashed lines represents the INTEGRAL/ISGRI window restricted to $17-150 \mathrm{keV}$, and the Fermi-LAT window restricted to $100 \mathrm{MeV}$ 6- $100 \mathrm{GeV}$. Right panels: trend between $\alpha_{X}$ vs $s$ (upper panel), and $\alpha_{\gamma}$ vs $s$ (lower panel), with $s$ ranging in [1.5-3.0], for SSC (green line), EC(purple line), and $\mathrm{SSC}+\mathrm{EC}($ blue line). The black thin line represents the theoretical expectation from standard synchrotron theory, the relative deviations of $\alpha_{X}$, and $\alpha_{\gamma}$ w.r.t. synchrotron expectations 6are reported in the bottom panel.

same parameters by the observed values of $\alpha_{\gamma}$ in the Fermi-LAT spectral window (in the case of HBLs) . To model the blazars SED, and to obtain trends among $s, \gamma_{\min }, \alpha_{X}$, and $\alpha_{\gamma}$ we use an accurate numerical code (Tramacere et al., 2009), deriving trends by looping over the parameters of interest.

\subsection{Linking $s$ to $\alpha_{X}$ and $\alpha_{\gamma}$}

The left panels of Fig. 1 show by an oblique dashed black line that, both in the case of FSRQs and I/LBLs (top panel), and HBLs (bottom panel), the rising part of the synchrotron component, and the rising part of the IC emission, have similar spectral slopes. We stress, that in the case of FSRQs and LBLs, the synchrotron self-absorption (red line, upper left panel) usually hides the spectral portion linked to $s$ (orange line, left upper panel), hence for this class of objects INTE$G R A L$ data represent a unique tool to infere this parameter. According to the standard synchrotron theory (Rybicki \& Lightman, 1979), the observed synchrotron photon index is linked to $s$ by the well known relation: $\alpha \simeq(s-1) / 2+1$. By showing that the value of $\alpha_{X}$ and $\alpha_{\gamma}$, observed in the IC emission, are close to the value of the photon index observed in the synchrotron spectrum range ruled by the spectral slope $s$, we demonstrate that we can use the $\alpha_{\gamma}$, and $\alpha_{X}$ to derive $s$, that is our 

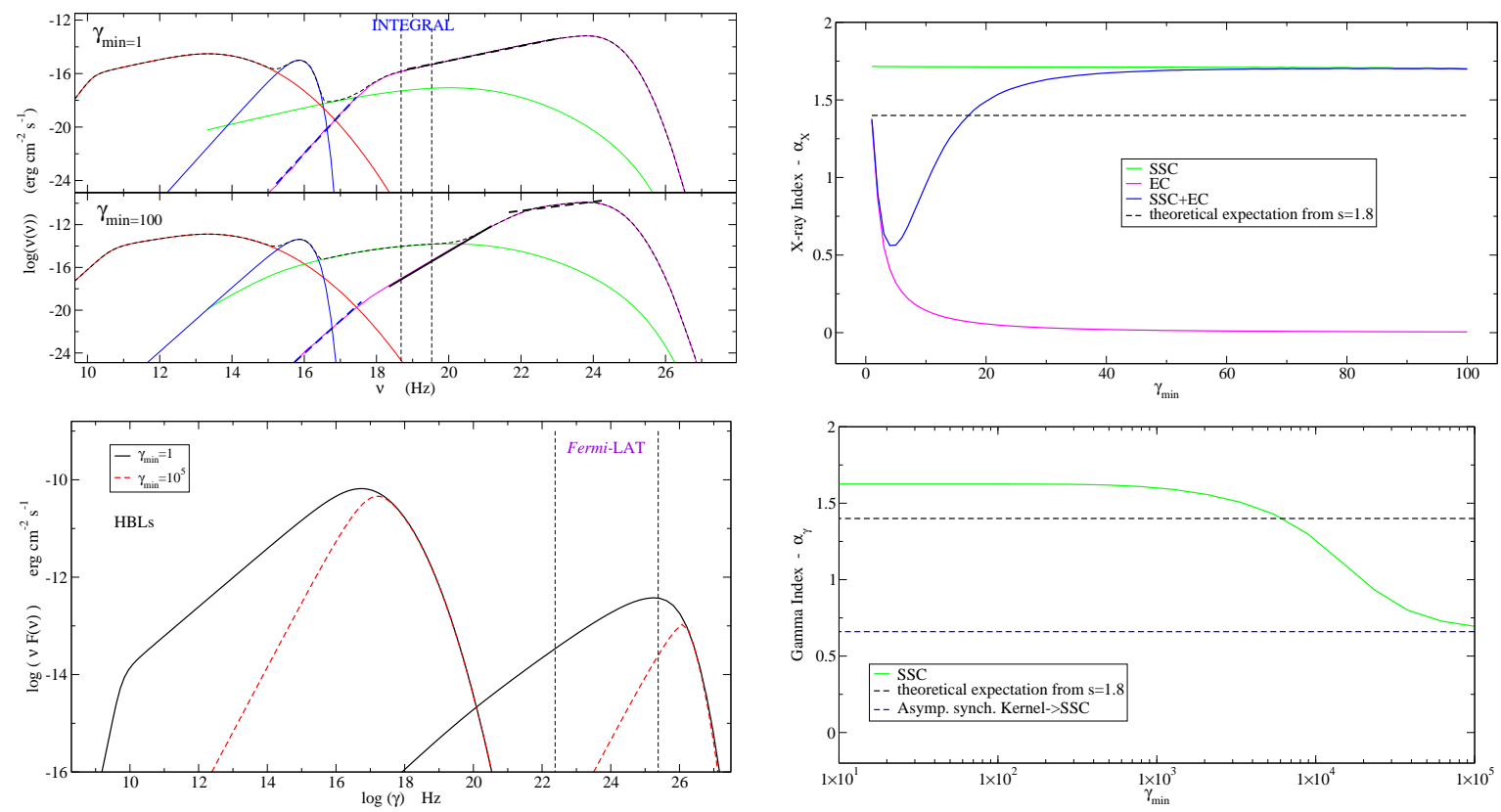

Figure 2: Left panels: Evolutions of the typical SEDs as a function of $\gamma_{\min }$, same color code as in left panels of Fig. 1. Upper panel shows the case of FSRQs and I/LBLs for $\gamma_{\min }=1$, and $\gamma_{\min }=100$, lower panel shows the case of HBLs for $\gamma_{\min }=1, \gamma_{\min }=1 \mathrm{e} 5$ (lowre panel). Right panels: the trend between $\alpha_{X}$ and $\gamma_{\min }$ (upper panel, case of FSRQs and IBLs/LBLs), and $\alpha_{\gamma}$ vs. $\gamma_{\min }$ (case of HBLs), fixing $s=1.8$ in both cases.

goal. The right panels of Fig. 1 show the trend of $\alpha_{X}$ vs $s$ (FSRQs, and I/LBLs, top panel), and $\alpha_{\gamma}$ vs $s$ (HBLs, bottom panel). We obtained $\alpha_{X}$ and $\alpha_{\gamma}$ by fitting the numerically computed SEDs in the $17-150 \mathrm{keV}$ band, for the case of INTEGRAL/ISGRI and in the $100 \mathrm{MeV}-100 \mathrm{GeV}$ band, for the case of Fermi-LAT. We computed numerical SEDs with $s$ ranging in [1.5-3.0]. In the case of FSRQs and I/LBLs (right top panel), both EC and EC+SSC values of $\alpha_{X}$ are close to the theoretical expectation from synchrotron theory (black line) within $\simeq 5 \%$. We note some deviation of the SSC+EC form the pure EC trend for $s<2$. This is due to the larger contribution from the SSC component when $s$ is harder. In the case of $\alpha_{\gamma}$ for HBLs, we get still a good correlation between $s$ and $\alpha_{\gamma}$, but $s$ values are systematically softer compared to synchrotron theory prediction, within $\simeq(15-20) \%$. We stress, that in the case of HBLs, a sampling of the rising part of the synchrotron component, is not easy, Indeed, it would require at least Optical and radio/mm data, and also the removing of the contamination from the galaxy contribution. Fermi-LAT data, hence gives values of $s$ with a lower bias when compared to those coming from Optical and radio/mm data.

\subsection{Linking $\gamma_{\min }$ to $\alpha_{\gamma}$ and $\alpha_{X}$}

To derive the value of $\gamma_{\min }$ we investigate the evolution of the shape of the rising part of the IC component as $\gamma_{\min }$ is increasing. For the classes of FSRQs and I/LBLs, we compare the two cases of $\gamma_{\min }=1$ and $\gamma_{\min }=100$ in the left upper panels of Fig. 2. For $\gamma_{\min }=1$ the rising part of the EC component presents two branches, the one indicated by the dashed blue line has the same photon index of the Rayleigh-Jeans region of the external thermal photon field. This branch falls typically below the ISGRI spectral window. There is a second branch, whose photon index is determined 
by the slope of the electron distribution (as discussed in the previous section) indicated by a black dotted line. This branch falls typically in the INTEGRAL/ISGRI spectral range. In the case of $\gamma_{\min }=100$, it is evident that the INTEGRAL window is not anymore dominated by the electron power law index, on the contrary $\alpha_{X}$ approaches asymptotically the value of 0 . In the right upper panel of Fig. 2 we show the trend of $\alpha_{X}$ vs $s$, obtained fitting our numerical SEDs. We plot the value of $\alpha_{X}$ as a function of $\gamma_{\min }$, fixing $s=1.8$. The expected values of $\alpha_{X}$, for the case of $\gamma_{\min }=100$ and for the $\mathrm{EC}$ and the $\mathrm{EC}+\mathrm{SSC}$ cases, are significantly different from the prediction for the case $\gamma_{\min }=1$, hence the INTEGRAL photon index offers a good signature to constrain the value of $\gamma_{\text {min }}$. We also stress, that the INTEGRAL window is quite far from the EC branch contaminated by the thermal external photon field, hence INTEGRAL data offer a better estimator for $s$ compared to X-ray detector operating in the typical $0.2-10 \mathrm{keV}$ band.

In the left lower panel of Fig. 2 we investigate the case of HBLs, by comparing the two scenarios of $\gamma_{\min }=1$ and $\gamma_{\min }=10^{5}$, both for the same value of $s=1.8$. In both the cases $\left(\gamma_{\min }=1\right.$ and $\gamma_{\min }=10^{5}$ ), we should observe in the power-law branch of the synchrotron and IC emission, the photon index determined by $s=1.8$. Actually,we get this value only in the case of $\gamma_{\min }=1$. This behavior has the following explanation. If we denote by $v_{S}(\gamma) \simeq 3.7 \times 10^{6} B \gamma^{2}(\mathrm{~Hz})$, the typical rest-frame energy emitted by a particle with a Lorentz factor of $\gamma$, interacting with a magnetic field of intensity $B$, and radiating synchrotron emission, we have that above $v_{S}\left(\gamma_{\min }\right)$, the emitted spectrum photon index will be determined by $s$, according to $\alpha \simeq(s-1) / 2+1$. Below $\gamma_{S}\left(\gamma_{\min }\right)$, the synchrotron spectrum will be described by the asymptotic low-energy approximation of the single particle synchrotron emission, that is a power law with the photon index $\alpha \simeq-3 / 4$ (Rybicki \& Lightman, 1979). In the right lower panel of Fig. 2, we plot the expected trend of $\alpha_{\gamma}$ vs $\gamma_{\text {min }}$, obtained by fitting the numerical SED in the Fermi-LAT spectral window, for the value of $s=1.8$. For values of $\gamma_{\min } \lesssim 10^{4}, \alpha_{\gamma}$ is close to the synchrotron expectation within $\simeq 15 \%$. For values of $\gamma_{\min }$ above $\simeq 10^{4}$, it approaches the the asymptotic low-energy approximation of the single particle synchrotron emission. This analysis, shows clearly, that the spectral shape in the Fermi-LAT window con be used as a good estimator of the value of $\gamma_{\min }$ in the case of HBLs.

\section{MonteCarlo approach and Comparison with observed statistics}

To compare our predicted trends with the observed data we use a MonteCarlo (MC) approach. Specifically, we generate both the SSC and EC numerical computation of the SEDs, extracting the input parameters of the models from a random distribution. This allows us to cover a larger volume of the parameter space, and to investigate how the trends, discussed above, changes when several parameters are changing simulataneously. In the case of FSRQs and I/LBLs, we make two MC runs, randomizing all the models parameters, and fixing $\gamma_{\min }=1$ and $\gamma_{\min }=100$ respectively. The corresponding results are summarized in the left panel of Fig. 3. The observed $\alpha_{X}$ distribution of INTEGRAL FSRQs and I/LBLs (red line) is clearly compatible with the case of $\gamma_{\min }=1$, (blue line) and rules out the case of $\gamma_{\min }=100$, both in the EC dominated scenario (green line) and in the $\mathrm{SSC}+\mathrm{EC}$ case (green dashed line). Moreover, from the range of the observed $\alpha_{X} \simeq[1.0-1.8]$ we estimate a corresponding range for $s \simeq[1.2-2.8]$. To study Fermi HBLs, we make two MC runs, fixing $\gamma_{\min }=1$, and $\gamma_{\min }=10^{4}$, respectively. The results are summarized in the right panel of Fig. 3. Also in this case, we are able to rule out the scenario with a large value of $\gamma_{\min }\left(\gamma_{\min }=10^{4}\right.$, 

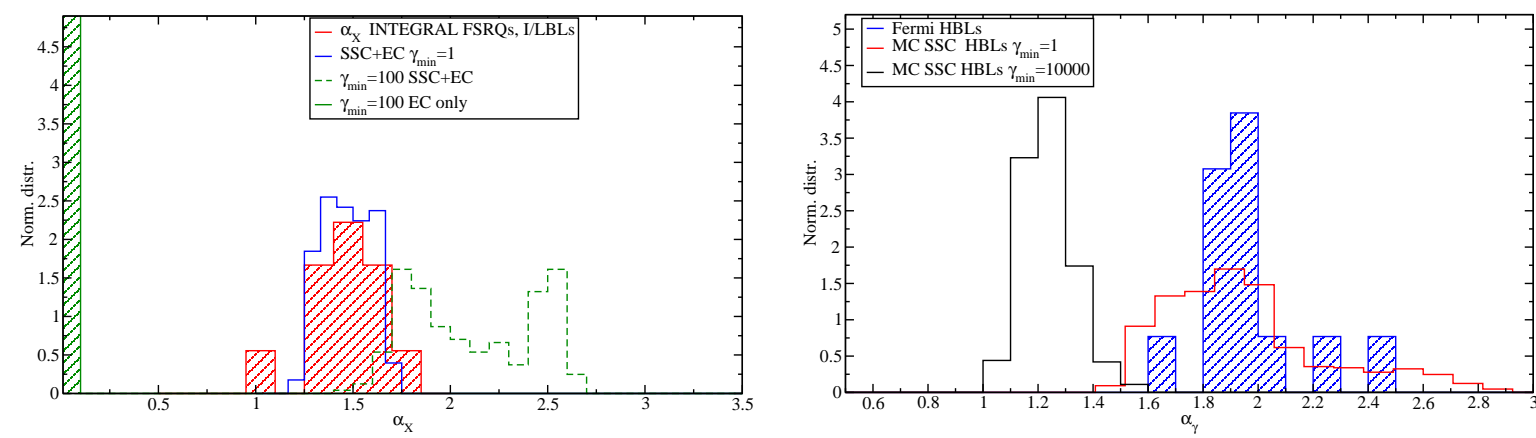

Figure 3: Histograms for MC runs for the FSRQs and I/LBLs compared to INTEGRAL observed $\alpha_{X}$ statistics (left panel), and for the HBLs compared to Fermi-LAT observed $\alpha_{\gamma}$ statistics (right panle).

black line). The case of $\gamma_{\min }=1$ shows a better matching with the observed Fermi values of $\alpha_{\gamma}$, and shows also the same drop in the histogram around the value of $\alpha_{\gamma} \simeq 2$. The observed range of $\alpha_{\gamma} \simeq$ [1.5-2.5] returns a range for $s \simeq$ [2.0-3.0], tacking into account, that in the case of the SSC scenario, as shown in Sect. 2.1, there is systematic softening of $s$ of about a $20 \%$, the fiducial range for $s$ should be $\simeq[1.6-2.4]$

\section{Conclusions}

We showed that is possible to use the values of $\alpha_{X}$ observed in INTEGRAL FSRQs and I/LBLs, and $\alpha_{\gamma}$ observed in Fermi HBLs, to constrain $\gamma_{\min }$ and $s$. According to our analysis, in the case of FSRQs and I/LBLs, INTEGRAL data rule out a scenario with $\gamma_{\min }>>1$. In the case of HBLs, Fermi data hints for a scenario with $\gamma_{\min }<<10^{4}$, in agreement with with previous analysis of the HBL object Mrk 421 based on simultaneous X-ray-to-UV data (Tramacere et al., 2009). The predicted range for $s$, derived from observed data, are $\simeq[1.6-2.4]$, and $\simeq[1.2-2.8]$, respectively for HBLs, and FSRQs and I/LBLs. The similarity in the range of the predicted values of $s$, and the same trend of the position of the low-energy cut-off of the electron distribution, observed in FSRQs and BL Lacs, are interesting features, hinting for a common acceleration scenario acting in the two classes of objects.

\section{References}

Abdo, A. A., Ackermann, M., Ajello, M., et al. 2010, The Astrophysical Journal, 715, 429

Dermer, C. D. \& Schlickeiser, R. 2002, ApJ, 575, 667

Ghisellini, G. \& Maraschi, L. 1989, ApJ, 340, 181

Jones, T. W., O’dell, S. L., \& Stein, W. A. 1974, ApJ, 188, 353

Padovani, P. \& Giommi, P. 1995, MNRAS, 277, 1477

Ricci, C., Walter, R., Courvoisier, T. J.-L., Paltani, S. 2011, A\&A in press, arXiv:1104.3676

Rybicki, G. B. \& Lightman, A. P. 1979, Radiative processes in astrophysics (New York, Wiley-Interscience, 1979. 393 p.)

Sikora, M., Begelman, M. C., \& Rees, M. J. 1994, ApJ, 421, 153

Sikora, M., Blazejowski, M., Moderski, R., \& Madejski, G. M. 2002, ApJ, 577, 78

Tramacere, A., Giommi, P., Perri, M., Verrecchia, F., \& Tosti, G. 2009, A\&A, 501, 879

Ubertini, P., Lebrun, F., Di Cocco, G., 2003, A\&A, 411, 131 